\title{
Minimally invasive bilateral decompressive lumbar laminectomy with unilateral approach: patient series
}

\author{
Megan M. Finneran, DO, ${ }^{1}$ Anant Naik, BS, ${ }^{2}$ John C. Hawkins, DO, ${ }^{1}$ and Emilio M. Nardone, MD ${ }^{1}$ \\ ${ }^{1}$ Department of Neurological Surgery, Carle BroMenn Medical Center, Normal, Illinois; and ${ }^{2}$ Carle Illinois College of Medicine, Champaign, Illinois
}

BACKGROUND Minimally invasive bilateral decompressive lumbar laminectomy with a unilateral approach is a less destructive procedure compared to the traditional open bilateral laminectomy. The objective of this study is to report the authors' experience with this technique. The first 26 cases performed using the unilateral approach for bilateral decompression are described. Baseline characteristics, operative time, blood loss, and intraoperative complications were collected retrospectively. No specific surgical equipment is needed for this technique.

OBSERVATIONS Twenty-six patients and a total of 40 lumbar levels were treated. Mean operative time was 82 minutes per level and mean estimated blood loss was $40.4 \mathrm{~mL}$ per level. Mean length of hospitalization was 1.65 days. Cerebrospinal fluid leak occurred in 1 of 26 (3.85\%) cases.

LESSONS Although improved stabilization needs to be proven in future long-term studies to clearly show a decrease in need for fusion, the initial experience with a unilateral approach is positive and continued use in minimally invasive spine surgery seems promising.

https://thejns.org/doi/abs/10.3171/CASE21676

KEYWORDS unilateral approach; minimally invasive; lumbar; laminectomy; stenosis

Decompressive lumbar laminectomy is a common spinal procedure performed for lumbar stenosis. Indications for surgical intervention include radiculopathy and neurogenic claudication in patients for whom conservative management has failed. ${ }^{1}$ The traditional laminectomy is performed in an open fashion in which the spinous process, lamina, ligaments, and medial facets are removed.

A laminectomy conducted from a unilateral approach focuses on functional decompression of stenosis while maintaining stabilization. It is predicted that sparing the posterior elements as well as the superior portion of the lamina decreases blood loss and hospital stay as well as long-term destabilization. ${ }^{2}$ The approach has been described utilizing a tubular system. We present a unique technique that uses a surgical microscope and emphasizes lateral rotation of the operative bed to maximize visualization while maintaining a minimally invasive approach.

\section{Study Description}

Data Collection

All patients treated with Current Procedural Terminology code 63047 in one community hospital were reviewed. Operative reports were examined, and 26 operations performed with a unilateral approach for bilateral decompression were included. Operative time, blood loss, and intraoperative complications were assessed. The institutional review board approved this study.

\section{Surgical Technique}

Patient Inclusion

A unilateral approach can be considered for single or multilevel lumbar stenosis between L2 and S1, below the level of the spinal cord. The authors have used the unilateral approach for up to four consecutive levels simultaneously, and it is used for cases of laminectomy only that do not include pedicle screw fixation.

\section{Side of Approach}

The authors choose the side most symptomatic in regard to subjective complaints, objective findings such as weakness, and/or most stenotic on imaging. Consideration should also be given to the angle of the spinous process. When the spinous process is angled in one direction rather than midline, approaching from 

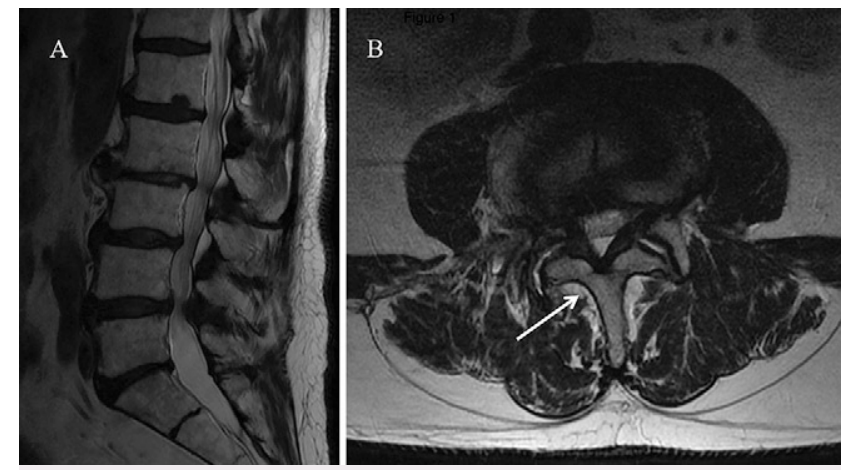

FIG. 1. A preoperative sagittal T2-weighted magnetic resonance image of the lumbar spine shows multilevel stenosis (A). An axial T2weighted image (B) at the level of $L 3-4$ shows a spinous process that is angled toward the left. This facilitates a right-side approach, as shown by the white arrow that points to the right hemilamina.

the contralateral side allows a more favorable corridor of access (Fig. 1).

When the patient presents with symptoms that are equal bilaterally and radiographic findings are equivocal, the approach is the surgeon's preference. It is our experience that a right-sided approach is technically more favorable for a right-handed surgeon and the opposite for a left-handed surgeon, particularly with regards to decompressing the contralateral lateral recess.

\section{Patient Positioning}

The patient is placed in prone position on a Wilson frame and meticulously secured to the frame and operating table with tape at multiple fixation points. These should include the gluteal region and hips as well as the mid-upper torso (Fig. 2). A safety strap will not be adequate. The authors wrap 3-inch silk tape around the patient and the table. Prior to sterile draping, the operating table should be airplaned (laterally rotated) to the right and left to ensure the patient is safely and adequately secured. Airplaning can be performed safely to a maximum of approximately 20 degrees in either direction.

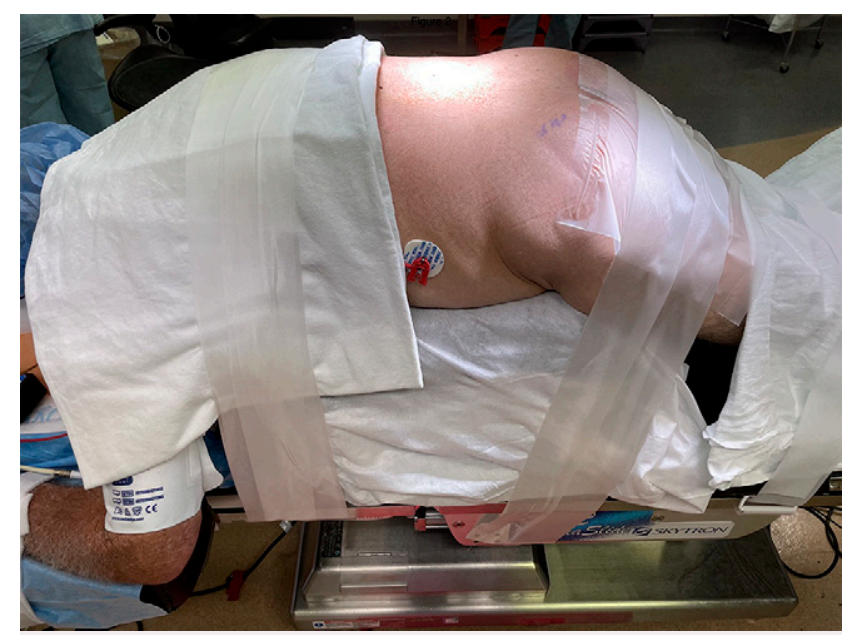

FIG. 2. The patient should be fastened to the operating bed with silk tape wrapped around the mid-upper torso and the gluteal region. Thorough fixation is essential to rotate the operating bed safely.

\section{Procedure}

An incision is made approximately $5 \mathrm{~mm}$ off the midline at the most lateral aspect of the spinous process. A length of $3 \mathrm{~cm}$ is adequate for single-level decompression. The fascia is opened at its attachment to the spinous process ipsilaterally, and the muscle disconnected on the side of the approach. The ipsilateral side of the spinous process and caudal aspect of the lamina is exposed, extending laterally to include the medial facet.

A narrow Versa-Trac retractor blade is placed laterally, and a pin placed medially, angled either caudally or cranially to avoid interference with visualization of the field to provide exposure. The retractor should be positioned so the instrumentation is away from the surgeon; this will allow an assistant to lift the retractor to improve visualization. Once the surgical level is confirmed with a lateral radiograph, bone work is initiated.

The authors favor a minimally invasive, self-irrigating Medtronic $3 \mathrm{~mm}$ electric burr. The caudal portion of the ipsilateral lamina and medial facet are eggshelled cranially to the level just above the attachment of the ligamentum flavum to achieve a functional decompression. Medially, the base of the spinous process is drilled to allow access to the contralateral side (Fig. 3). As the medial drilling progresses, the patient is airplaned away from the surgeon in small increments. When the patient is rotated away from the surgeon, an assistant should lift the retractor device, holding it in place to effectively rotate the view of the retractors toward the surgeon to improve contralateral visualization.

Once the ipsilateral drilling is complete, the midline of the ligamentum flavum can be identified. The contralateral lamina slopes deep laterally as it connects to the contralateral facet. The underlying ligament can be followed as the lamina is eggshelled and the ligament falls away toward the contralateral facet. The drilling is then carried out to the contralateral medial facet, with special attention to the caudal and cephalad corners. The bone work on the contralateral side should primarily be performed with the drill; the steep downward angle of the lamina is difficult to remove with a Kerrison.

When drilling is complete, the patient is rotated back to midline and Kerrison punches are used to remove the final layer of bone

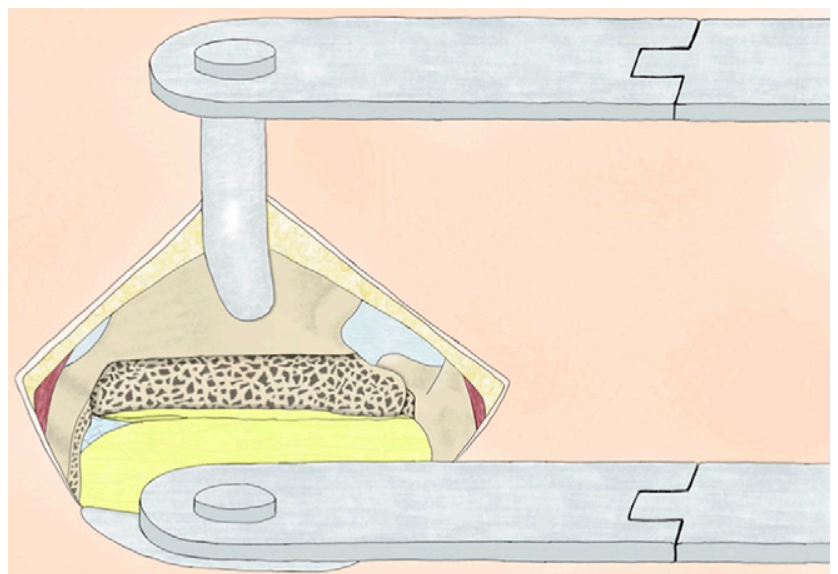

FIG. 3. The ipsilateral lamina and medial facet are drilled to a level just caudal to the attachment of the ligamentum flavum. The caudal aspect of the lamina is spared to minimize destabilization. Bone work is carried out medially to the base of the spinous process to allow access to the contralateral side. 

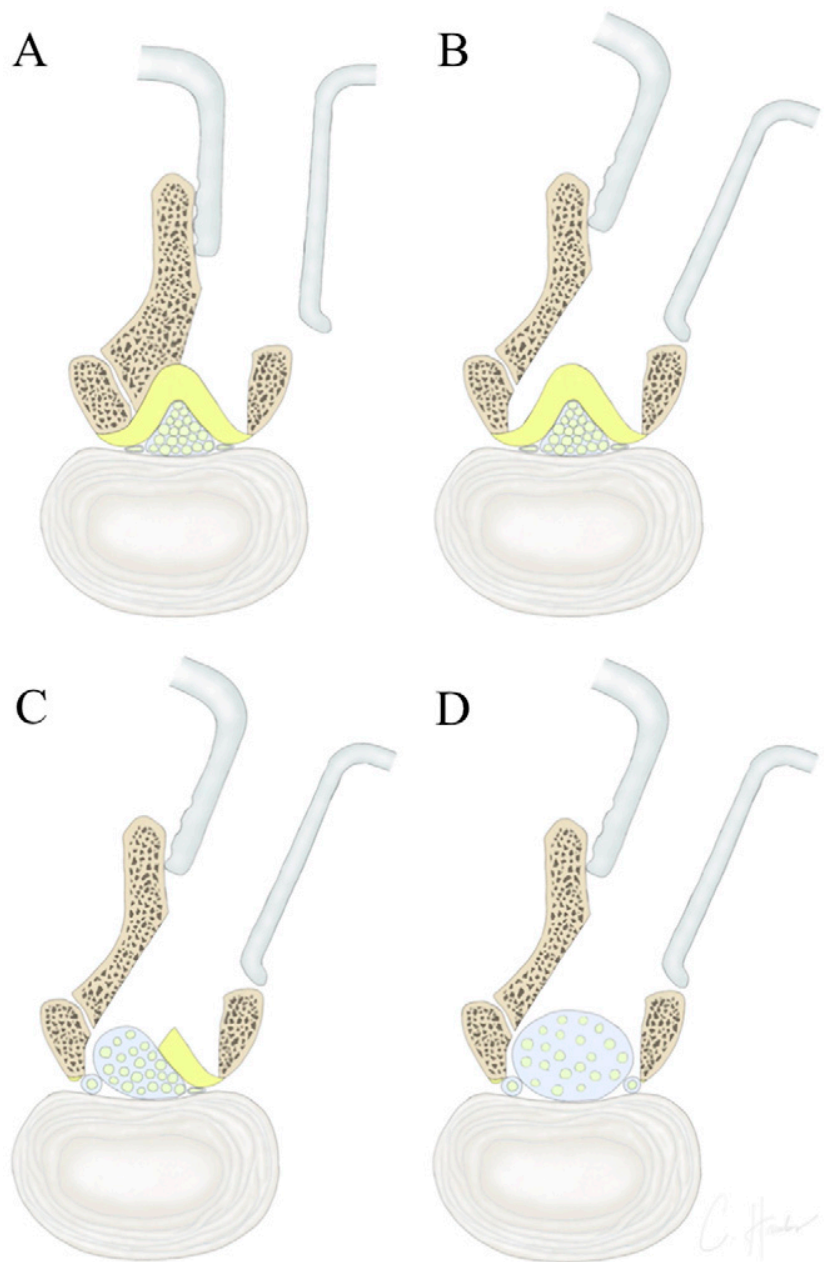

FIG. 4. The ipsilateral lamina, medial facet, and base of the spinous process are removed with the drill and Kerrison punches $(\mathbf{A})$. The patient is then rotated away from the surgeon and an assistant lifts the retractor to allow removal of the contralateral bone in a similar fashion (B). The ligament is removed bilaterally (C) until the decompression is completed (D).

on the ipsilateral side (Fig. 4A). This is continued until the ligamentum flavum is fully exposed, including the cephalad attachment. The patient is airplaned away from the surgeon and the work is continued contralaterally (Fig. 4B). Once the bone work is complete, the ligamentum is removed bilaterally until a complete decompression, extending to the superior aspect of the lamina one level caudally, has been achieved (Fig. 4C and D).

It is essential to complete the majority, if not all, of each step prior to progressing to the next step to facilitate the work and minimize risks. In fact, it is challenging to drill contralaterally if the ipsilateral dura has already been exposed. Once the decompression is completed, meticulous hemostasis is performed. For a one-level decompression a drain is usually not used. On the contrary, a 10F Jackson-Pratt drain is laid for multilevel laminectomies. Fascia is closed with 0-Vicryl, subcutaneous tissue with 2-0 Vicryl, and skin with 4-0 Vicryl in a subcuticular fashion. Steri-strips and a large adhesive bandage are then applied. Staples may be used for skin with multilevel laminectomies when the incision is greater than $5 \mathrm{~cm}$.
Results

Twenty-six patients were operated on using unilateral approach for bilateral decompression between July 2013 and December 2015. Eleven patients were male and 15 were female with an average age of 66.3 years. Fifteen patients presented with main complaint of bilateral lower extremity pain, three with right lower extremity pain, five with left lower extremity pain, two with bilateral lower extremity weakness, and one with bilateral lower extremity numbness.

Fifteen cases were approached from the left side and 11 from the right. Mean operative time was 126.2 minutes for each operation and 82.0 minutes per operative level. Estimated blood loss was an average of $62.1 \mathrm{~mL}$ per operation and $40.4 \mathrm{~mL}$ per surgical level. Mean length of stay for all patients was 1.65 days. There was one $(3.85 \%)$ intraoperative complication in the form of cerebrospinal fluid leak.

\section{Discussion}

Lumbar spinal stenosis (LSS) is a common cause of back pain, particularly in elderly patients. It leads to chronic pain and disability, which in turn results in a diminished quality of life, mobility, and overall function. ${ }^{3}$ Whereas large-scale meta-analyses for the management of LSS have not concluded the superiority of surgical intervention over conservative therapy, ${ }^{3}$ the rates of decompressive surgical procedures have increased. ${ }^{4}$ This has driven the need for strategies that reduce surgical complications and improve patient outcomes for laminectomy procedures.

\section{Observations}

We describe a minimally invasive unilateral approach for bilateral decompressive laminectomy with the aim to mitigate complications in the short and long term. Open bilateral laminectomy is the conventional strategy for the management of LSS. In this approach, both sides of the lamina as well as the spinous process of the targeted vertebrae are removed. While relatively rare, complications such as leakage of cerebrospinal fluid, hematoma, or infection may occur. ${ }^{5}$

Various methods of unilateral approach have been described. Hwa Eum et al. ${ }^{6}$ described an endoscopic method with two portal incisions made laterally, one for the endoscope and the other for instruments with acceptable results, but two other studies found no advantage over traditional laminectomy. ${ }^{7,8}$ Phan et al. ${ }^{9}$ outlined a minimally invasive unilateral approach through the use of tubular retractors with similar improvements of patient-centered outcomes, improvement in postoperative mobility, and decreased use of opioids for postoperative pain when compared to open laminectomy. 10,11

The efficacy of a unilateral approach for bilateral decompression has been investigated but its superiority remains to be clearly established. Studies have analyzed outcomes after conventional open lumbar laminectomy compared to microendoscopic laminectomy and found lower rates of postoperative complications, surgical site infections, and postoperative delirium. ${ }^{12}$ The microendoscopic approach also demonstrated a greater reduction in back pain and patient satisfaction than the traditional open laminectomy. Furthermore, the length of hospital stay was significantly reduced for minimally invasive surgical patients. ${ }^{12,13}$

The most common long-term complication of conventional laminectomy is the need for reoperation, which may be accelerated by destabilization of the posterior elements. Reoperation may consist of laminectomy at an adjacent level for acquired adjacent segment disease or an instrumented fusion for spondylolisthesis. In one 
retrospective analysis of 500 first-time traditional, open bilateral laminectomies, the reoperation rate was found to be $14.4 \%$ over a mean of 3.4 years. ${ }^{5}$ Of these, $55.56 \%$ of reoperation surgeries consisted of decompression alone and $44.44 \%$ underwent decompression and fusion.

It is postulated that by preserving the posterior elements and superior aspect of the lamina, a unilateral functional approach should decrease the risk of destabilization and therefore decrease the rate of reoperation. One meta-analysis of 2,496 patients that evaluated reoperation rates after laminectomy, either open or minimally invasive, found an overall reoperation for instability rate of $1.8 \%$ and a postoperative radiographic instability rate of $5.5 \% .{ }^{14}$ Reoperation rates were higher among those treated with open laminectomy $(4.1 \%){ }^{14}$

\section{Lessons}

One argument against the unilateral approach, both anecdotally and in the literature, is an increase in operative time..$^{12,13}$ This can particularly be true when first learning the technique; there is undoubtedly a learning curve. However, this approach uses the microscope and Versa-Trac retractors and does not require mastery of new tools. The authors have used the unilateral microsurgical method described here in a teaching institution for more than 10 years with successful results by neurological surgery residents. Further research is needed to demonstrate a clear advantage of this method over traditional open laminectomy.

Patients with normal to overweight body habitus (body mass index less than $30.0 \mathrm{~kg} / \mathrm{m}^{2}$ ) and those with no prior surgery at the proposed operative level are considered favorable cases for the described approach, particularly when first learning the technique. Additionally, a more vertical lamina may facilitate the approach to the contralateral side. Although less favorable, obese patients and those with a more flat-sloped lamina can still be treated with this approach. Major facet hypertrophy does not represent a significant obstacle to properly perform this procedure and in fact is a common finding in patients harboring moderate to severe lumbar stenosis. The only absolute contraindication the authors have identified is previous same level surgery.

The unique technique described in this manuscript encompasses a unilateral approach to achieve bilateral decompression for lumbar laminectomy. This method carries the advantage of a minimally invasive approach without use of tubular devices or endoscope. The authors predict it is superior to the traditional open bilateral approach, most notably with regards to preservation of the posterior elements and maintenance of stabilization. Further investigation is needed to discover if the method truly reduces the risk of reoperation.

\section{References}

1. Costa F, Sassi M, Cardia A, et al. Degenerative lumbar spinal stenosis: analysis of results in a series of 374 patients treated with unilateral laminotomy for bilateral microdecompression. J Neurosurg Spine. 2007;7(6):579-586.

2. Rahman M, Summers LE, Richter B, Mimran RI, Jacob RP. Comparison of techniques for decompressive lumbar laminectomy: the minimally invasive versus the "classic" open approach. Minim Invasive Neurosurg. 2008;51(2):100-105.
3. Zaina F, Tomkins-Lane C, Carragee E, Negrini S. Surgical versus non-surgical treatment for lumbar spinal stenosis. Cochrane Database Syst Rev. 2016;2016(1):CD010264.

4. Deyo RA, Mirza SK, Martin BI, Kreuter W, Goodman DC, Jarvik JG. Trends, major medical complications, and charges associated with surgery for lumbar spinal stenosis in older adults. JAMA. 2010; 303(13):1259-1265.

5. Bydon M, Macki M, Abt NB, et al. Clinical and surgical outcomes after lumbar laminectomy: an analysis of 500 patients. Surg Neurol Int. 2015;6(4)(suppl 4):S190-S193.

6. Hwa Eum J, Hwa Heo D, Son SK, Park CK. Percutaneous biportal endoscopic decompression for lumbar spinal stenosis: a technical note and preliminary clinical results. J Neurosurg Spine. 2016;24(4): 602-607.

7. Park SM, Park J, Jang HS, et al. Biportal endoscopic versus microscopic lumbar decompressive laminectomy in patients with spinal stenosis: a randomized controlled trial. Spine J. 2020;20(2): 156-165.

8. Chiu RG, Patel S, Zhu A, Aguilar E, Mehta Al. Endoscopic versus open laminectomy for lumbar spinal stenosis: an international, multiinstitutional analysis of outcomes and adverse events. Global Spine J. 2020;10(6):720-728.

9. Phan K, Teng I, Schultz K, Mobbs RJ. Treatment of lumbar spinal stenosis by microscopic unilateral laminectomy for bilateral decompression: a technical note. Orthop Surg. 2017;9(2):241-246.

10. Mobbs R, Phan K. Minimally invasive unilateral laminectomy for bilateral decompression. JBJS Essential Surg Tech. 2017;7(1):e9.

11. Mobbs RJ, Li J, Sivabalan P, Raley D, Rao PJ. Outcomes after decompressive laminectomy for lumbar spinal stenosis: comparison between minimally invasive unilateral laminectomy for bilateral decompression and open laminectomy: clinical article. J Neurosurg Spine. 2014;21(2):179-186.

12. Oichi T, Oshima Y, Chikuda $\mathrm{H}$, et al. In-hospital complication rate following microendoscopic versus open lumbar laminectomy: a propensity score-matched analysis. Spine J. 2018;18(10):1815-1821.

13. Phan K, Mobbs RJ. Minimally invasive versus open laminectomy for lumbar stenosis: a systematic review and meta-analysis. Spine (Phila Pa 1976). 2016;41(2):E91-E100.

14. Guha D, Heary RF, Shamji MF. latrogenic spondylolisthesis following laminectomy for degenerative lumbar stenosis: systematic review and current concepts. Neurosurg Focus. 2015;39(4):E9.

\section{Disclosures}

The authors report no conflict of interest concerning the materials or methods used in this study or the findings specified in this paper.

\section{Author Contributions}

Conception and design: Finneran, Nardone. Acquisition of data: Finneran, Nardone. Analysis and interpretation of data: Finneran, Naik. Drafting the article: Finneran, Naik, Hawkins. Critically revising the article: Finneran, Nardone. Reviewed submitted version of manuscript: Finneran, Nardone. Approved the final version of the manuscript on behalf of all authors: Finneran. Statistical analysis: Finneran. Administrative/technical/material support: Finneran, Nardone. Study supervision: Nardone.

\section{Correspondence}

Megan M. Finneran: Carle BroMenn Medical Center, Normal, IL. megan.finneran7@gmail.com. 